\title{
Cloud motion and magnetic fields: Four clouds in the Cepheus Flare region (Corrigendum)
}

\author{
E. Sharma ${ }^{1,2} \odot$, G. Maheswar ${ }^{1}$, and S. Dib $^{3} \odot$ \\ ${ }^{1}$ Indian Institute of Astrophysics (IIA), Sarjapur Road, Koramangala, Bangalore 560034, India \\ e-mail: ektasharma.astro@gmail.com \\ 2 Department of Physics and Astrophysics, University of Delhi, Delhi 110007, India \\ ${ }^{3}$ Max Planck Institute for Astronomy, Königstuhl 17, 69117, Heidelberg, Germany
}

A\&A, 658, A55 (2022), https://doi.org/10.1051/0004-6361/202140495

Key words. ISM: magnetic fields - ISM: structure - polarization - techniques: polarimetric - errata, addenda

An error occurred during the production process. The names of authors Ekta Sharma and Sami Dib were incorrectly written. They are corrected in this corrigendum. 